\title{
Descending necrotizing mediastinitis due to an odontogenic infection: A case report

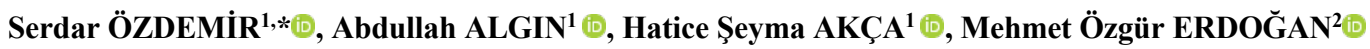 \\ ${ }^{1}$ Department of Emergency Medicine, University of Health Sciences Umraniye Training and Research Hospital, Istanbul, Turkey ${ }^{2}$ Department of Emergency Medicine, Bahcesehir University, Istanbul, Turkey
}

\begin{tabular}{l} 
Received: $18.02 .2021 \quad$ Accepted/Published Online: 24.02 .2021 \\
\hline Abstract \\
Descending necrotizing mediastinitis a clinical entity formed by the spreading of cervical infection from the dental and oropharyngeal structures \\
through the deep cavities between the deep fascia on the neck to the mediastinum, pleural and pericardial spaces with necrosis of soft tissue and \\
has a high mortality. Herein we present the case of a 73 -year-old admitted to emergency department with septic clinic. The patient was \\
diagnosed with descending necrotizing mediastinitis due to odontogenic infection. Although sternal irrigation, sternal debridement and \\
mediastinal drainage were performed patient was died postoperative third day.
\end{tabular}

Keywords: case report, descending necrotizing mediastinitis, mediastinitis, mediastinum,

\section{Introduction}

Mediastinum is the part between the two lungs that is located medially in the thorax. Mediastinitis is an inflammation of the mediastinal tissue, which can be quite fatal. Most acute mediastinitis can develop secondary to esophageal perforation or chest trauma. Descending necrotizing mediastinitis (DNM) is a polymicrobial infection disease caused by the spread of oropharyngeal flora to the mediastinum. The infection spreads through the deep fascia around the neck into the mediastinum, the pleural and pericardial cavities, and even into the abdomen. It can cause empyema, pericardial and pleural effusion, peritonitis, cardiac tamponade, and intrathoracic bleeding. Mortality has been reported to be between $4.3-40 \%$ (1). In this case report, we aimed to present a case of DNM which is rare and has high mortality.

\section{Case Report}

A 73 years old male patient admitted to our department with fever and altered mental status for five days. He had undergone extractions of the right mandibular second molar teeth 20 days before presentation. He had history of an untreated infection on left mandibular third molar teeth for a week. He had a sinus tachycardia of $110 /$ minute, blood pressure $70 / 40 \mathrm{mmHg}$, and fever $39^{\circ} \mathrm{C}$. Glasgow Coma Scale score of the patient was 13 (V:4, E:3, M:6). He had severe submandibular and neck (extending to chest) non-fluctuant swelling, that was warm to palpation and erythematous. On examination, cervical subcutaneous crackling, crunching sound could be heard with stethoscope over the precordium during systole were noted (Fig. 1). His complete blood count revealed leukocyte of $27.8 \mathrm{~K} / \mathrm{uL}$, hemoglobin of $12.6 \mathrm{~g} / \mathrm{dl}$, hematocrit of $36.2 \%$, and platelets of $120,000 \mathrm{nl}$. Biochemical parameters were within the normal ranges. CT scan was obtained, which showed free air in the pericardial space, anterior mediastinum, and subcutaneous tissue of the neck, bilateral pleural effusion and compressive atelectasis (Fig. 2). From the clinical examination and CT findings it was noted compatible with necrotizing mediastinitis.

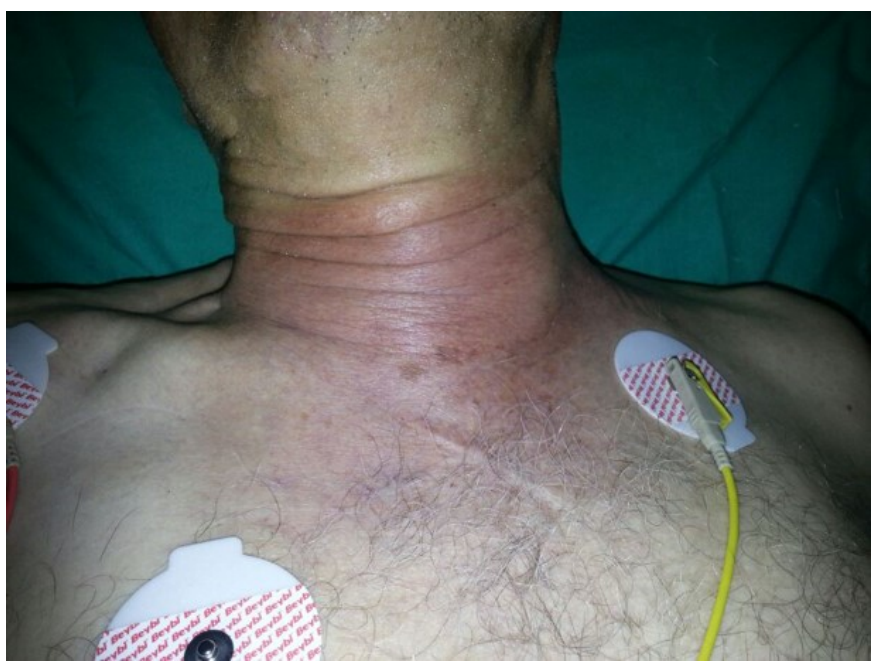

Fig. 1. Erythematous appearance of submandibular area and neck

Empirically, he was given parenteral antibiotic therapy that consisted of intravenously (IV) administered metronidazole $500 \mathrm{mg}$ eight hourly and IV administered ceftriaxone $1 \mathrm{gm} 12$ hourly). Endotracheal intubation was performed and, severe edema of the vocal cords was noted, on direct laryngoscopy. He was transferred to operating room urgently where he underwent sternal irrigation, sternal debridement and mediastinal drainage. Patient was admitted 


\section{Özdemir et al. / J Exp Clin Med}

to surgical intensive care unit (ICU) post operatively while hemodynamically unstable (blood pressure 62/47) $\mathrm{mmHg}$, and afebrile $\left(36.7{ }^{\circ} \mathrm{C}\right)$. He was sedated, mechanically ventilated in ICU. Vancomycin $1000 \mathrm{mg}$ and levofloxacin $750 \mathrm{mg}$ were added to antibiotherapy by intensive care unit team. His postoperative period was essentially unremarkable without showing significant relief of symptoms and vital parameters. During ICU follow-up, he was hypotensive and tachycardic despite inotrope support. On the third postoperative day he developed sudden onset of cardiac arrest from which he could not be resuscitated.

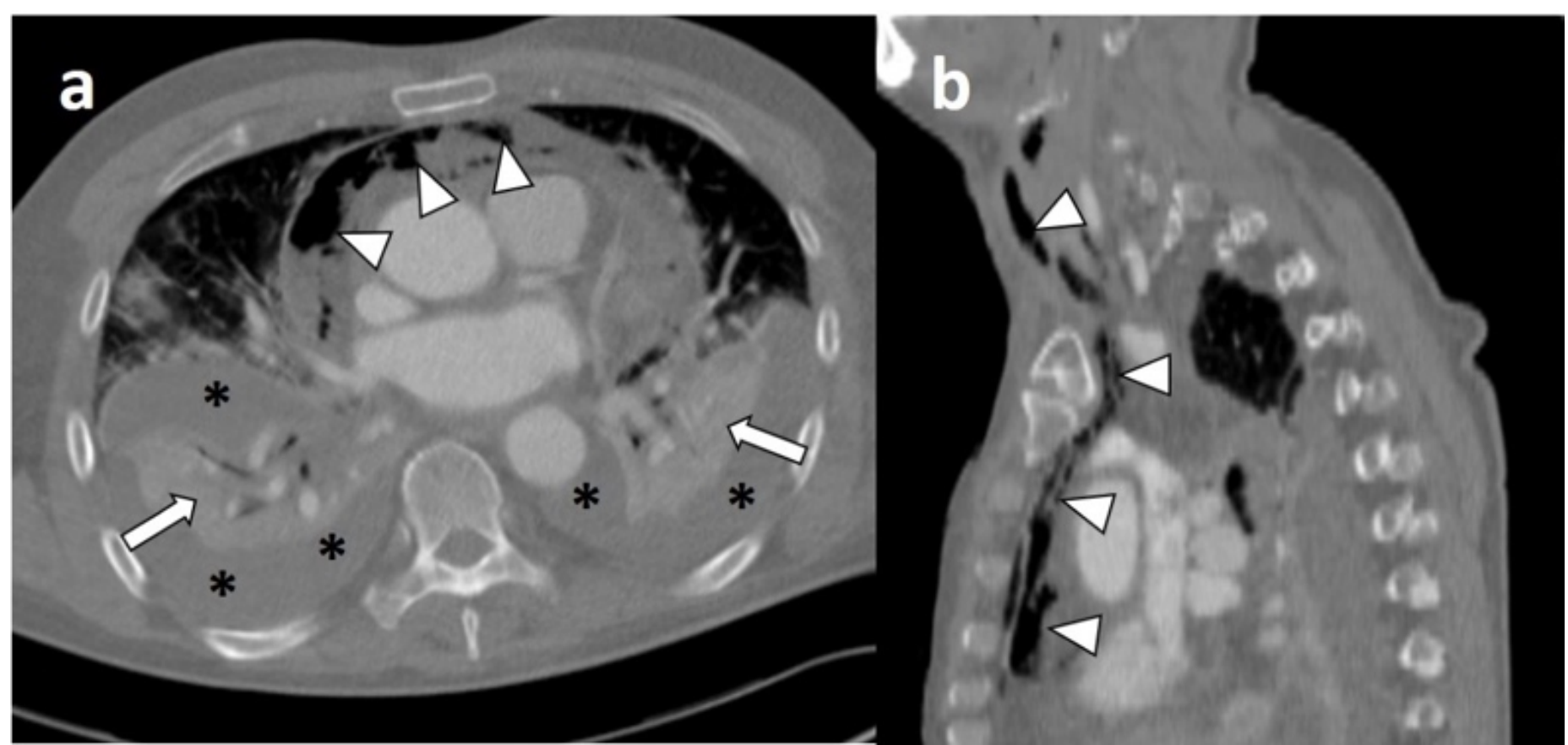

Fig. 2. The transverse and sagittal reformatted contrast enhanced thorax CT images of the patient. Bilateral pleural effusion (asterisks) and compressive atelectasis (arrows) are observed. Free air is seen in the pericardial space, anterior mediastinum, and subcutaneous tissue of the neck (arrowheads) are noted compatible with necrotizing mediastinitis

\section{Discussion}

Mediastinitis is a rare but severe infection of the mediastinum. The most common cause of acute mediastinitis is esophageal perforation $(90 \%)$. Secondary causes are spread of lung and pleural infections into the mediastinum and lymphohematogenous spread. The reaching of the oropharyngeal or cervical infection to the mediastinum through the deep facial plan is a rare cause of acute mediastinitis and is called descending mediastinitis (2). DNM is a clinical entity formed by the spreading of cervical infection from the dental and oropharyngeal structures through the deep cavities between the deep fascia on the neck to the mediastinum, pleural and pericardial spaces with necrosis of soft tissue and has a high mortality of $4.3-40 \%$ (1). In order of frequency, odontogenic, pharyngeal and cervical infections can be the causes of DNM (2). Pathogenesis of DNM was first described in the literature by Pearse in 1938 (3). DNM is classified in three types; Type I, Type IIa and Type IIb. Type I is local infection, Type IIa is diffuse mediastinitis, spreading into the lower anterior mediastinum and Type IIb is diffuse mediastinitis, spreading into both the anterior and posterior lower mediastinum (4). Our case was Type IIa DNM because mediastinitis was diffuse and extending into the lower anterior mediastinum not posterior lower mediastinum.

Symptoms of DNM aren't specific. Symptoms depend on the etiology of disease and consist of infective process causing tachycardia and fever also altered mental status in late cases as in our case. Sepsis and septic shock often develop abruptly, and symptoms may be related to it. There is no specific laboratory finding. Infection markers such as procalcitonin, leucocyte count, and C-reactive protein are often increased. Thrombocytopenia could be predictor of severe sepsis.

The preferred radiological imaging method contrastenhanced CT of the neck and thorax. High-resolution computed tomography (HRCT) can assist in diagnosis and can be used for diagnosis. Contrast-enhanced CT help identifying the etiology of the mediastinitis and planning potential surgical interventions $(5,6)$.

Management of DNM depends on management of severe infection, sepsis and septic shock and the underlying reason. Patients with DNM should manage in ICU, because there is relationship between mortality and late ICU admission and the high ICU severity scores on admission to ICU (7).

DNM is typically polymicrobial infection. Empirical antimicrobial therapy of DNM, should cover anaerobic and aerobic bacteria commonly associated with odontogenic and pharyngeal infections. A combination of a third-generation cephalosporin such as ceftriaxone with metronidazole or a combination of clindamycin and piperacillin/tazobactam are 
recommended for empirical antimicrobial therapy in DNM patients $(8,9)$.

Surgical treatment is important to control infection and debridement of affected tissue. Cervical and mediastinal drainage is classically required for DNM (2). In our case sternal irrigation, sternal debridement and mediastinal drainage was performed.

In conclusion, DNM, is rare entity and a life-threatening infection. Symptoms and laboratory findings are non-specific. Early empirical antimicrobial treatment and surgical treatment significantly associated morbidity and mortality.

\section{Conflict of interest}

We declare no conflict of interest.

\section{Acknowledgments}

We received no financial support for the research, authorship, or publication of this article. We asked the patient to help us to publish the case report in an international journal for discussion, including disease symptoms, diagnosis, and image related content. The patient agreed us to use his medical records and signed the consent form.

\section{References}

1. Deu-Martín M, Saez-Barba M, Sanz IL, Peñarrocha RA, Vielva LR, Montserrat MR. Mortality risk factors in descending necrotising mediastinitis. Arch Bronconeumol. 2010;46(4):182-7.

2. Pastene B, Cassir N, Tankel J, Einav S, Fournier PE, Thomas P, Leone M. Mediastinitis in the intensive care unit patient: a narrative review. Clin Microbiol Infect. 2020;26(1):26-34.

3. Pearse HE. Mediastinitis Following Cervical Suppuration. Ann Surg 1938;108(4):588-611.

4. Endo S, Murayama F, Hasegawa T, Yamamoto S, Yamaguchi T, Sohara $Y$, et al. Guideline of surgical management based on diffusion of descending necrotizing mediastinitis Jpn J Thorac Cardiovasc Surg. 1999;47(1):14-9.

5. Exarhos DN, Malagari K, Tsatalou EG, Benakis SV, Peppas C, Kotanidou A, et al. Acute mediastinitis: spectrum of computed tomography findings. Eur Radiol. 2005;15(8):1569-74.

6. B Erdogan, MO Erdogan, S Colak, O Kibici, K Bozan, B Alper. An isolated hyoid bone fracture caused by blunt trauma to the neck. J Pak Med Assoc. 2015;65 (11): 1233-4.

7. Palma DM, Giuliano S, Cracchiolo AN, Falcone M, Ceccarelli G, Tetamo R, et al. Clinical features cases. Infection. 2016;44 (1):77-84.

8. Prado-Calleros HM, Jiménez-Fuentes E, Jiménez-Escobar I. Descending necrotizing mediastinitis: Systematic review on its treatment in the last 6 years, 75 years after its description. Head Neck 2016;38(Supp 1): E2275-83.

9. Gunaratne DA, Tseros EA, Hasan Z, Kudpaje AS, Suruliraj A, Smith MC, et al. Cervical necrotizing fasciitis: Systematic review and analysis of 1235 reported cases from the literature. Head Neck. 2018;40(9):2094-102. 